\title{
Relative intensity noise transfer in high-order distributed amplification through ultra-long fiber cavities
}

\author{
Mercedes Alcón-Camas, ${ }^{1}$ Juan Diego Ania-Castañón ${ }^{2, *}$ \\ ${ }^{1}$ Photonics Research Group, School of Engineering and Applied Science, Aston University, \\ Aston Triangle, Birmingham, B4 7ET, UK \\ ${ }^{2}$ Instituto de Óptica "Daza de Valdés", CSIC, C/ Serrano 121, 28006 Madrid, Spain \\ *juan.diego@io.cfmac.csic.es
}

\begin{abstract}
Among the different possible amplification solutions offered by Raman scattering in optical fibers, ultra-long Raman lasers are particularly promising as they can provide quasi-losless second order amplification with reduced complexity, displaying excellent potential in the design of low-noise long-distance communication systems. Still, some of their advantages can be partially offset by the transfer of relative intensity noise from the pump sources and cavity-generated Stokes to the transmitted signal. In this paper we study the effect of ultra-long cavity design (length, pumping, grating reflectivity) on the transfer of RIN to the signal, demonstrating how the impact of noise can be greatly reduced by carefully choosing appropriate cavity parameters depending on the intended application of the system.
\end{abstract}

Ultra-long Raman fiber lasers, Raman amplification, RIN transfer, optical communications

\section{Introduction}

The advantages of distributed optical fiber Raman amplifiers [1,2] over traditional lumped optical amplification are well known, and include an improved noise performance and extended bandwidth. Standard conventional optical fibers are susceptible to Raman scattering, which can be used to provide Raman amplification without restriction to a particular range of wavelengths. This brings the opportunity of upgrading existing transmission systems through the implementation of Raman amplification, on its own or combined with other existing amplification solutions, increasing system performance and capacity. Recently, ultra-long Raman fiber lasers (URFLs) [3-7], in which transmission fiber acts as an ultra-long laser cavity that is used for signal amplification, have been shown to be able to further reduce signal power excursion, improving noise performance and presenting itself as an interesting alternative to high-order Raman amplification schemes.

Among the challenges of distributed Raman amplification, an important one is relative intensity noise (RIN) [8] transfer. This is a pump-to-signal noise transfer with origin in the intensity fluctuations of the pump laser source. Any fluctuation on the pump power will be reflected in a variation of signal gain, and thus be imprinted in the signal, creating an additional source of noise that, in some situations and with a low-quality pump source, can be as damaging a noise source as amplified spontaneous emission (ASE) itself.

The main contribution of this paper is the development of a numerical model for higher order distributed Raman amplifiers, in particular but not in exclusivity for ultra-long Raman fiber lasers. This numerical model allows us to investigate the effect of features design, such as span length, pump distribution and fiber Bragg gratings (FBGs) reflectivity, on the relative intensity noise transfer performance of the system.

Photonics North 2010, edited by Henry P. Schriemer, Rafael N. Kleiman, Proc. of SPIE Vol. 7750 , $775017 \cdot$ ? 2010 SPIE · CCC code: 0277-786X/10/\$18 - doi: 10.1117/12.872861 


\section{Theoretical model}

In order to describe our numerical model, we propose to study two particular Raman amplifier systems. They are schematically described in Figure 1. The first system corresponds to a bi-directionally pumped URFL, with pumps at $1366 \mathrm{~nm}$. It involves a conventional standard optical fiber confined between a pair of high reflectivity FBGs with a central wavelength of $1455 \mathrm{~nm}$, corresponding to the first Raman Stokes of the pump source. The optical fiber length considered for this work ranges from 1 to $100 \mathrm{~km}$. The pump power at $1366 \mathrm{~nm}$ is coupled into the system through WDMs. The wavelength of the transmitted signal is at $1550 \mathrm{~nm}$, corresponding to the second Stokes of the pump source.

As the pump power injected at $1366 \mathrm{~nm}$ grows above the lasing threshold for the fiber cavity, a first Stokes component around $1455 \mathrm{~nm}$ is generated and trapped between the grating reflectors. This component will behave as a second pump source, providing a wide Raman gain bandwidth to the transmitted signal, located around the second Stokes wavelength of $1550 \mathrm{~nm}$. This second-order cascading pumping structure provides reduced power excursion, and therefore, the system can provide quasi-lossless transparency around $1550 \mathrm{~nm}$ for a particular range of pump powers.

The second distributed Raman amplifier system involves a conventional standard optical fiber, ranging from 1 to $100 \mathrm{~km}$ similarly to the first system, but no reflectors at all. The optical fiber is bi-directionally pumped at the wavelengths $1366 \mathrm{~nm}$ and $1455 \mathrm{~nm}$. The pump powers from both pump sources are coupled into the system through WDMs. The transmitted signal is, as before, at $1550 \mathrm{~nm}$, corresponding to the second Stokes of the pump source at $1366 \mathrm{~nm}$ and the first Stokes of the pump source at $1455 \mathrm{~nm}$.

The way to operate this amplifier system will be as follows. We increase the pump power at $1366 \mathrm{~nm}$ to the same level as it would be done within the same conditions of span length, pump distribution and type of fiber for the first system. Therefore, the power profile at $1366 \mathrm{~nm}$ along the span length will be the same as the first system. Then, we calculate the initial power at $1455 \mathrm{~nm}$ at each end of the fiber span for our first system and use exactly the same $1455 \mathrm{~nm}$ power value for the pumps of our second system. This allows us to generate an equivalent amplifier system to the first one with exactly the same power distribution for $1366 \mathrm{~nm}, 1455 \mathrm{~nm}$ and the transmission wavelength $1550 \mathrm{~nm}$, but using an extra bi-directional pump source at $1455 \mathrm{~nm}$ instead of using FBGs with the same center of wavelength. Hence, average power distributions inside the two amplifiers are identical, which will make it possible to perform a direct comparison of their respective RIN transfer performances.

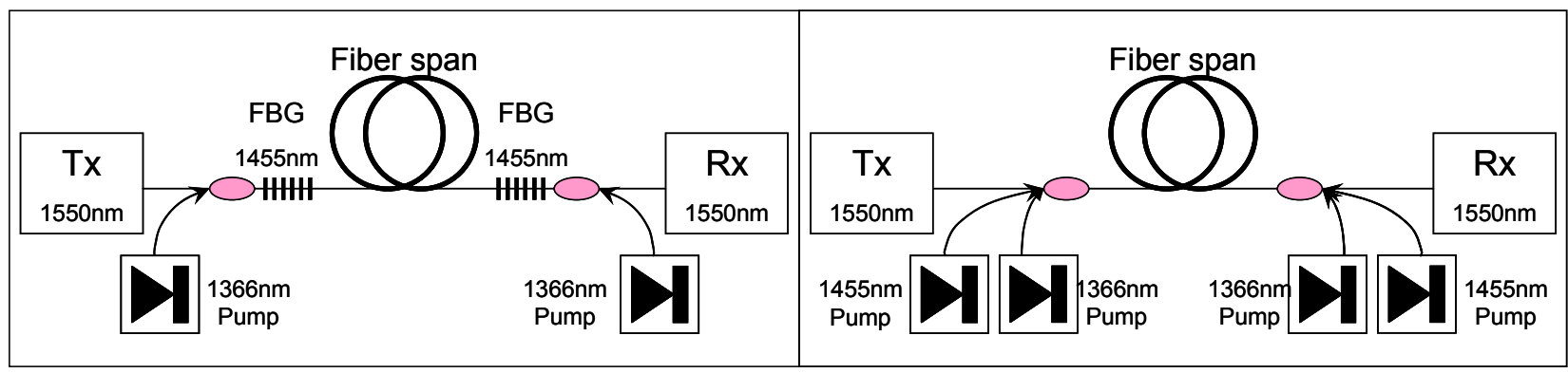

(a)

(b)

Figure 1: Schematic description of the studied bi-directional pumped Raman amplifier systems. (a) Ultra-long Raman fiber laser. (b) Equivalent power distribution system with dual pumping scheme.

As the power distribution should be the same for both systems given the same span length, pump power distribution and type of fiber, any difference on the RIN transfer values between these two systems is expected to come from the impact of having a laser cavity in the system instead of a dual wavelength pumping scheme. 
Therefore we can quantify how the pump-noise transfer, with origin in the intensity fluctuation of the laser source due to spontaneous emission, evolves and it is magnified when using a laser cavity as amplifier.

The ordinary differential equations that describe mathematically our model are subdivided in two groups. On one side we have the set of equations 1 to 5 describing the average power evolution of pumps, signal and ASE noise along the length of the fiber for a second order Raman amplifier. These set of equations take in account all relevant effects, including pump depletion, ASE and double Rayleigh scattering (DRS) noise. A more extensive description of the average power performance for our first system can be found in reference [4].

$$
\begin{aligned}
\frac{d P_{P 1}^{ \pm}}{d z}=\mp & \alpha_{1} P_{P 1}^{ \pm} \mp g_{1} \frac{v_{1}}{v_{2}} P_{P 1}^{ \pm}\left(P_{P 2}^{+}+P_{P 2}^{-}+4 h v_{2} \Delta v_{2}\left(1+\frac{1}{e^{h\left(v_{1}-v_{2}\right) / K_{B} T}-1}\right)\right) \pm \varepsilon_{1} P_{P 1}^{\mp} \\
\frac{d P_{P 2}^{ \pm}}{d z}=\mp & \alpha_{2} P_{P 2}^{ \pm} \pm g_{1}\left(P_{P 2}^{ \pm}+2 h v_{2} \Delta v_{2}\left(1+\frac{1}{e^{h\left(v_{1}-v_{2}\right) / K_{B} T}-1}\right)\right)\left(P_{P 1}^{+}+P_{P 1}^{-}\right) \\
& \mp g_{2} \frac{v_{2}}{v_{S}} P_{P 2}^{ \pm}\left(P_{S}+N_{S}^{+}+N_{S}^{-}+4 h v_{S} \Delta v_{S}\left(1+\frac{1}{e^{h\left(v_{2}-v_{S}\right) / K_{B} T}-1}\right)\right) \pm \varepsilon_{2} P_{P 2}^{\mp}
\end{aligned}
$$

$\frac{d P_{S}}{d z}=-\alpha_{S} P_{S}+g_{2} P_{S}\left(P_{P 2}^{+}+P_{P 2}^{-}\right)$

$\frac{d N_{S}^{+}}{d z}=-\alpha_{S} N_{S}^{+}+g_{2}\left(N_{S}^{+}+2 h v_{2} \Delta v_{2}\left(1+\frac{1}{e^{h\left(v_{2}-v_{S}\right) / K_{B} T}-1}\right)\right)\left(P_{P 2}^{+}+P_{P 2}^{-}\right)+\varepsilon_{S} N_{S}^{-}$

$\frac{d N_{S}^{-}}{d z}=\alpha_{S} N_{S}^{-}-g_{2}\left(N_{S}^{-}+2 h v_{2} \Delta v_{S}\left(1+\frac{1}{e^{h\left(v_{2}-v_{S}\right) / K_{B} T}-1}\right)\right)\left(P_{P 2}^{+}+P_{P 2}^{-}\right)-\varepsilon_{S}\left(P_{S}+N_{S}^{+}\right)$

On the other hand, a different set of ODEs, equations 6 to 8 , describe mathematically the evolution of the spectral densities of the amplitude noise along the span for a second order Raman amplification system. The cavity design parameters are introduced in the boundary conditions.

The subscripts 1,2 , and $\mathrm{S}$ denote the primary pump at $1366 \mathrm{~nm}$, secondary pump at $1455 \mathrm{~nm}$ and signal at $1550 \mathrm{~nm}$ respectively.

$$
\begin{aligned}
& \frac{d n_{1}^{ \pm}}{d z}+i d_{1}^{ \pm} \omega n_{1}^{ \pm}=\mp \alpha_{1} n_{1}^{ \pm} \mp g_{1} \frac{v_{1}}{v_{2}} P_{1}^{ \pm}\left(n_{2}^{+}+n_{2}^{-}\right) \mp g_{1} \frac{v_{1}}{v_{2}}\left(P_{2}^{+}+P_{2}^{-}\right) n_{1}^{ \pm} \pm \varepsilon_{1} n_{1}^{\mp} \\
& \frac{d n_{2}^{ \pm}}{d z}+i d_{2}^{ \pm} \omega n_{2}^{ \pm}=\mp \alpha_{2} n_{2}^{ \pm} \pm g_{1} P_{2}^{ \pm}\left(n_{1}^{+}+n_{1}^{-}\right) \pm g_{1} n_{2}^{ \pm}\left(P_{1}^{+}+P_{1}^{-}\right) \mp g_{2} \frac{v_{2}}{v_{S}} P_{2}^{ \pm} n_{S} \mp g_{2} \frac{v_{2}}{v_{S}} n_{2}^{ \pm} P_{S} \pm \varepsilon_{2} n_{2}^{\mp} \\
& \frac{d n_{S}}{d z}=-\alpha_{S} n_{S}+g_{2} P_{S}\left(n_{2}^{+}+n_{2}^{-}\right)+g_{2} n_{S}\left(P_{2}^{+}+P_{2}^{-}\right)+\varepsilon_{S} n_{S}^{-}
\end{aligned}
$$

Where $\mathrm{z}$ is the length measured along the fiber span. The group velocity is described by $v_{i}$, referring to the wavelength of the pump for subscript "1", first stokes for subscript "2", or signal for subscript "s". The attenuation coefficient, $\alpha$, is in units of $\mathrm{km}^{-1}$. The optical frequency, $v$, refers to the first order pump, second order, or signal. The strength of the coupling between the pump and the signal is determined by $g$, the Raman gain efficiency of the 
fiber which has units of (W Km) ${ }^{-1}$. The pump modulation frequency is described by $\omega$. The signs \pm correspond to the pumps propagating in $\pm \mathrm{z}$ direction, meaning forward $(+)$ and backward (-) propagating optical powers at the first order pump (1), second order pump (2) and signal (S). Here and through this paper is assumed that the signal propagates in the forward $+\mathrm{z}$ direction. $\varepsilon$ are the Rayleigh backscattering coefficients of the fiber at each particular frequency. The two FBGs for the Stokes wavelength are located at $\mathrm{z}=0$ and $\mathrm{z}=\mathrm{L}$ and have reflectivities R1 and R2 respectively. They are described by the two sets of boundary conditions:

For scheme 1

$P_{P 1}^{+}(0)=P_{10}^{+} ; P_{P 1}^{-}(L)=P_{10}^{-} ; P_{P 2}^{+}(0)=R_{1} P_{P 2}^{-}(0) ; P_{P 2}^{-}(L)=R_{2} P_{P 2}^{+}(L) ; N_{S}^{+}(0)=N_{0} ; N_{S}^{-}(L)=0 ; P_{S}(0)=P_{I N}$ $n_{1}^{+}(0)=n_{10} ; n_{1}^{-}(0)=n_{20} ; n_{2}^{+}(0)=R_{1} n_{2}^{-}(0) ; n_{2}^{-}(L)=R_{2} n_{2}^{+}(L) ; n_{S}(0)=0$

For scheme 2

$P_{P 1}^{+}(0)=P_{10}^{+} ; P_{P 1}^{-}(L)=P_{10}^{-} ; P_{P 2}^{+}(0)=P_{20}^{+} ; P_{P 2}^{-}(L)=P_{20}^{-} ; N_{S}^{+}(0)=N_{0} ; N_{S}^{-}(L)=0 ; P_{S}(0)=P_{I N}$ $n_{1}^{+}(0)=n_{10}^{+} ; n_{1}^{-}(0)=n_{10}^{-} ; n_{2}^{+}(0)=n_{20}^{+} ; n_{2}^{-}(L)=n_{20}^{-} ; n_{S}(0)=0$

The fiber parameters used in the simulation results for both systems, previously described by Figure 1 (a) and (b), are given in Table 1 and it corresponds to typical values for conventional SMF.

Table 1: Fiber parameters used in the simulation results

\begin{tabular}{cccc}
\hline Wavelength $(\mathrm{nm})$ & $g\left(\mathrm{~W}^{-1} \mathrm{~km}^{-1}\right)$ & $\alpha(\mathrm{dB} / \mathrm{km})$ & $\varepsilon\left(\mathrm{km}^{-1}\right)$ \\
\hline 1365 & 0.51 & 0.33 & $1.0 \times 10^{-5}$ \\
1455 & 0.36 & 0.26 & $6.0 \times 10^{-5}$ \\
1550 & - & 0.2 & $4.3 \times 10^{-5}$ \\
\hline
\end{tabular}

Although the two sets of equations $1-5$ and $6-8$ describe a second order Raman amplification system, in principle there is no limit for applying a similar mathematical approach to higher order Raman amplification systems, at the cost of making the solution of the system substantially more complex.

\section{Results and discussion}

The solution of the numerical simulation described mathematically by the set of equations 1 to 8 can yield the impact of the span length, pump power distribution, FBGs reflectivity and type of fiber on the RIN transfer of the optical Raman amplifier system. As clarification, all the calculations for the numerical simulation work shown on this manuscript are conditioned to the quasi-lossless condition unless otherwise stated. The quasi-lossless condition aims to achieve the lowest power variation along the length of the fiber regardless the signal power, span length, pump power distribution or any other parameter of the amplifier system.

The numerical results for the RIN transfer value at several span lengths are shown on Figure 2. The results are graphically represented for the two amplifier systems described previously, Figure 2 (a) the ultra-long laser cavity 
with $90 \%$ reflectivity FBGs and Figure 2 (b) the dual wavelength pumping scheme with no FBGs. The value of transmitted signal is $1 \mathrm{~mW}$ in both cases. The initial RIN value of the laser pump is $1 \mathrm{E}-9$. The span length ranges from 1 to $100 \mathrm{~km}$. The only variable parameter is the length of the fiber span; otherwise the pump power distribution is symmetric and the quasi-lossless condition is satisfied.

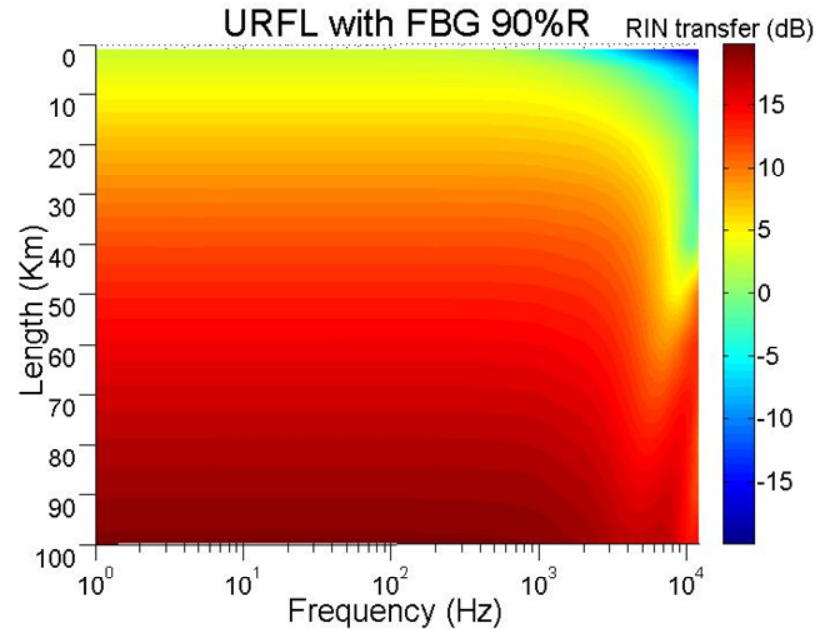

(a)

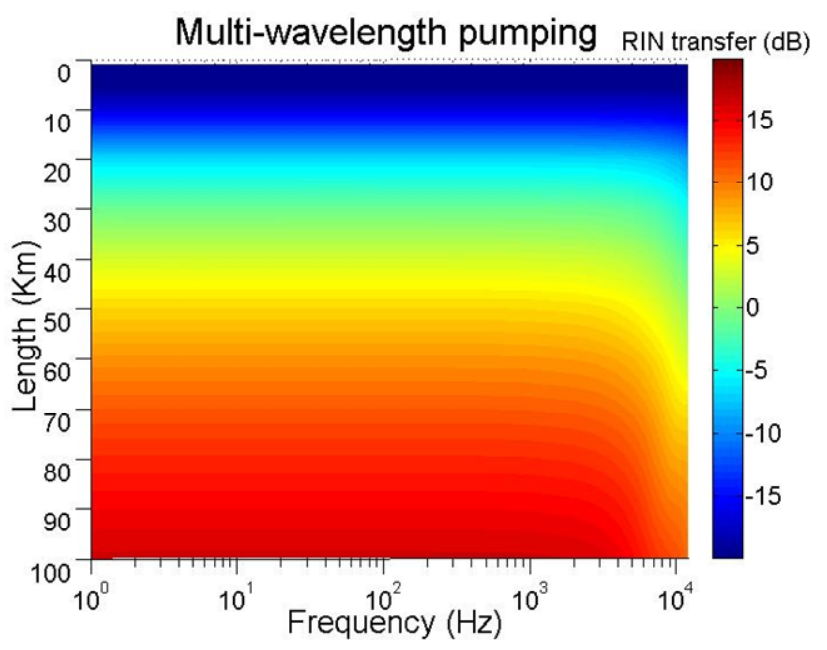

(b)

Figure 2: RIN transfer value at several span lengths for (a) laser cavity with $90 \%$ FBGs and (b) fiber span dual pumping scheme without reflectors

As a general trend, the maximum RIN transfer function value increases with length regardless of whether the Raman amplifier is a laser cavity or a second-order multi-wavelength pumped fiber span without reflectors. As an example, the maximum RIN value at $75 \mathrm{~km}$, for the cases of laser cavity with FBGs at $90 \%$ reflectivity and fiber span without reflectors, are $17 \mathrm{~dB}$ and $12.25 \mathrm{~dB}$ respectively, i.e. RIN transfer is $4.75 \mathrm{~dB}$ higher in the ultra-long cavity configuration. Although the difference exists for all cases, it reduces as the span length increases, and for 100 $\mathrm{km}$ it is below $3.5 \mathrm{~dB}$. A major difference between both systems results is the decline of the RIN transfer value at longer modulation frequencies. Whilst in the case of the dual pumping scheme without reflectors the RIN transfer drops gradually with increasing modulation frequency; in the case of the laser cavity the RIN transfer value shows a fluctuating behavior towards lower values, especially for the longer span lengths. Furthermore, as Figure 3 shows, the drop frequency, taken as $2 \mathrm{~dB}$ lower than the maximum value, is lower for the laser cavity case than the fiber span without reflectors, given the same fiber length and power conditions. This cut-off frequency value is not fixed for all the span length cases.

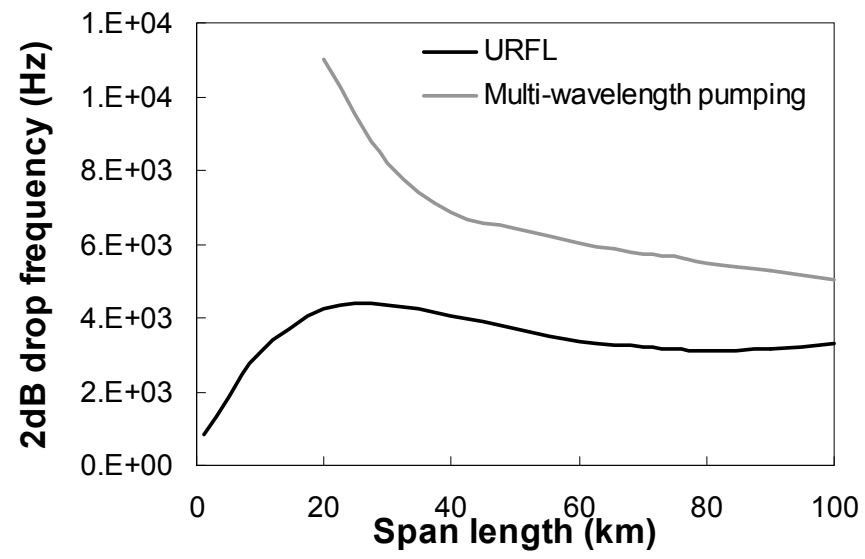

Figure 3: 2dB drop frequency versus span length. Comparison between two amplifier systems; a laser cavity with $90 \%$ reflectors and a fiber span dual pumping scheme without reflectors 
The effect of the pump distribution is shown on Figure 4 for the cases of (a) laser cavity with $90 \%$ reflectivity FBGs and (b) dual wavelength pumping scheme without reflectors. The value of transmitted signal is again $1 \mathrm{~mW}$ in both cases and the initial RIN of the laser pump is $1 \mathrm{E}-9$. The span length is fixed at $75 \mathrm{~km}$. The pump ratio is calculated as the forward pump divided by the total amount of pump (forward and backward).

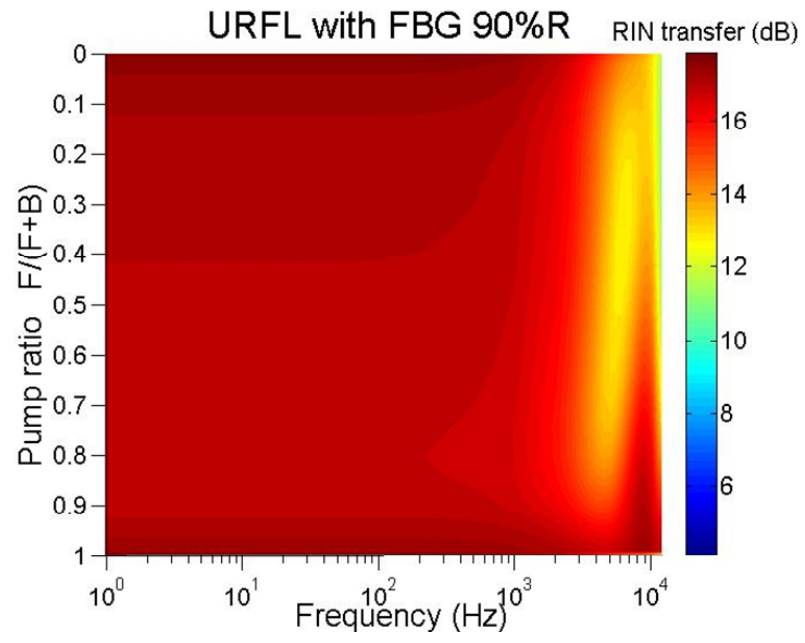

(a)

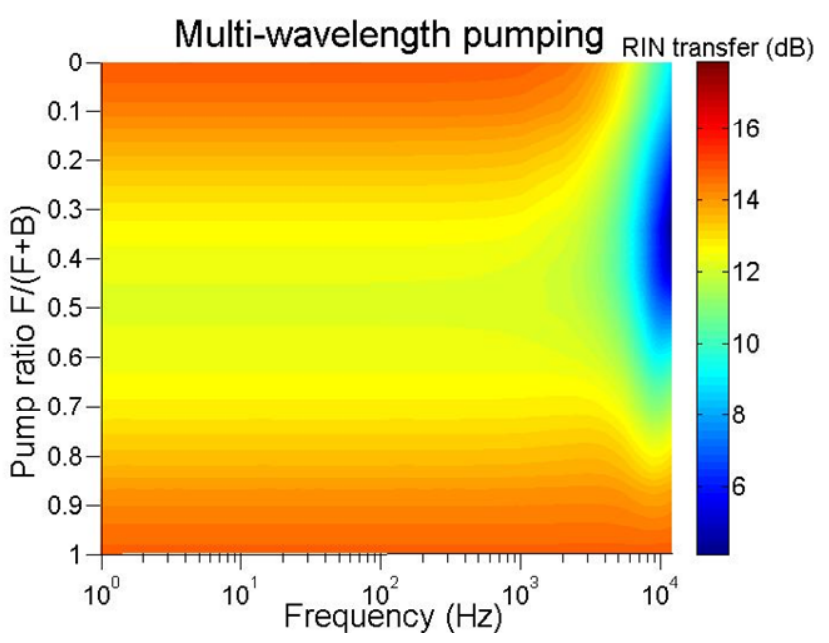

(b)

Figure 4: RIN transfer value for pumping distribution in the case of (a) $75 \mathrm{~km}$ laser cavity with $90 \%$ reflectors and (b) $75 \mathrm{~km}$ dualwavelength second-order pumping scheme without reflectors.

The pump power distribution has a clear impact on the RIN transfer value. For the case of a laser cavity, Figure 4 (a) the optimum pumping distribution for the lowest RIN transfer value is $80 \%$ forward pump and $20 \%$ backward pump, with a value of $16.83 \mathrm{~dB}$. Using any pump power distribution other than $80 \%$ forward $/ 20 \%$ backward will have a relative low variation, maximum $0.9 \mathrm{~dB}$, on the RIN transfer value. The case of dual pumping scheme without reflectors, Figure 4 (b) shows symmetric pumping to be the best option, with a maximum RIN transfer value of $12.25 \mathrm{~dB}$. The variation of the RIN transfer value for any other pump distribution other than symmetric pumping can be up to $2.6 \mathrm{~dB}$ for the conditions used on this work.

The profile of the RIN transfer value drops continuously for longer modulation frequencies in all the cases where the major pump contribution is from backward pumping (pump ratio towards 0 ). Forward pumping introduces a modulation of the RIN transfer value at longer frequencies, especially in the case of a laser cavity, displacing the drop frequency towards longer distances in the dual-wavelength pumping configuration. As we saw previously the drop frequency appears at shorter frequencies in the case of a laser cavity than the dual pumping scheme without reflectors, and therefore this modulation of the RIN transfer seems stronger for the case of laser cavity. For example, in a laser cavity case at $90 \%$ forward pump contribution, the peak fluctuation at longer modulation frequencies (i.e. $8.5 \mathrm{kHz}$ ) is slightly higher, up to $0.8 \%$, than at lower modulation frequency.

Figure 5 shows the RIN transfer values in a $75 \mathrm{~km}$ ultra-long laser cavity as grating reflectivity is varied $0.1 \%$ to $100 \%$. As previously, these results were calculated using $1 \mathrm{~mW}$ forward transmitted signal and $1 \mathrm{E}-9$ as initial pump RIN value of the laser pump. The main result is that increasing FBGs reflectivity does not implies always the 
highest value for RIN transfer; in fact the worst case is for relative low FBGs reflectivity, between 10 to $20 \%$ reflectivity.

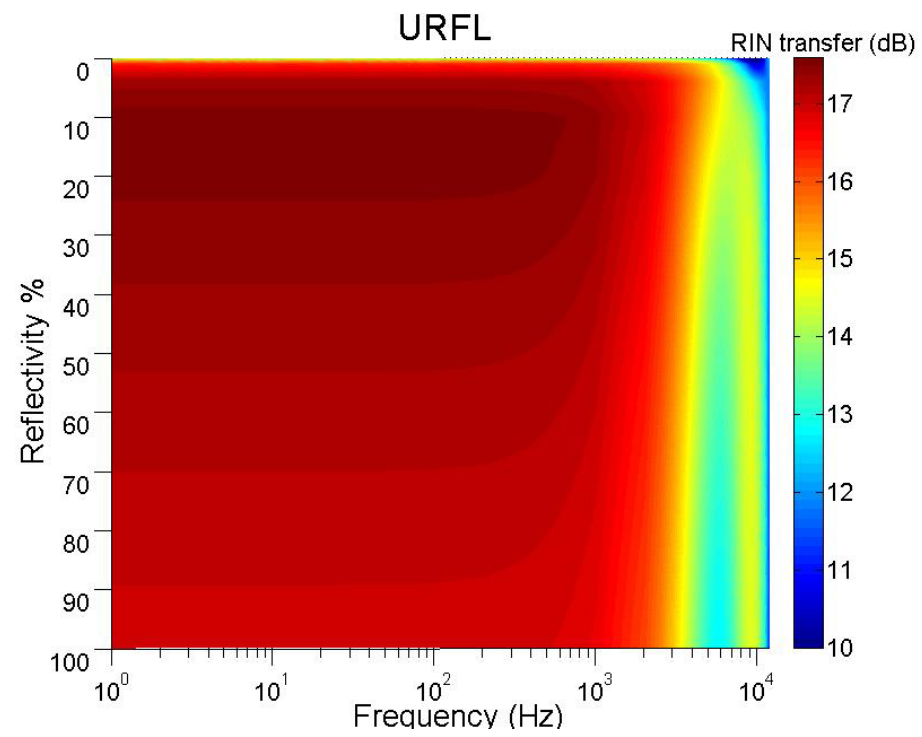

Figure 5: RIN transfer value for a $75 \mathrm{~km}$ laser cavity for different $\mathrm{FBG}$ reflectivities.

As observed on the previous results, the $2 \mathrm{~dB}$ drop frequency is lower for higher reflectivies, with the worst case of RIN transfer taking place at $15 \%$ reflectivity at low modulation frequencies, and can be as low as to $5 \%$ for higher frequencies of the order of $6 \mathrm{kHz}$.

\section{Conclusion}

We have described a numerical model for RIN transfer in higher order distributed Raman amplifiers, with special application to ultra-long Raman fiber lasers (URFL). This numerical model allows us a better understanding of how the design parameters of the laser cavity have an effect upon the RIN transfer performance of the system.

As expected the span length has a major impact on RIN transfer with an increasing RIN transfer value for longer lengths. At low modulation frequencies when transfer is usually highest, this RIN transfer value tends to converge towards a common value for long lengths, regardless of the rest of the design features of the Raman amplifier scheme. The maximum RIN transfer value is not linked to the highest reflectivity of the laser cavity, but in fact, happens at relative low reflectivity, $15 \%$.

The pump ratio is a key parameter with effects in two aspects of the RIN transfer. On the one side it affects the maximum RIN transfer itself, although in different degrees depending of whether we are using a laser cavity or a dual-wavelength pumping scheme without reflectors. In addition, it also affects the modulation of the RIN transfer function at higher frequencies. This modulation is stronger at higher frequencies as the contribution of the forward pumping increases. A higher contribution of the backward pumping leads to a faster drop of the RIN transfer value with increasing frequency.

\section{References}

1. M. N. Islam, "Raman amplifiers for telecommunications", IEEE J. Select. Topics in Quantum Electron., 8 (3), pp. 548-559 (2002) 
2. J. Bromage, "Raman amplification for fiber communications systems," J. of Lightwave Tech. 22(1) 79-93 (2004)

3. J.D. Ania-Castañón, T.J. Ellingham, R. Ibbotson, X. Chen, L. Zhang, S.K. Turitsyn, "Ultralong Raman Fiber Lasers as virtually lossless optical media," Phys. Rev. Lett. 96(2), 023902 (2006)

4. J.D. Ania-Castañón, Quasi-lossless transmission using second-order Raman amplification and fibre Bragg gratings," Opt. Express 12(9), pp. $4372-4377$ (2004)

5. A.E. El-Taher, J.D. Ania-Castañón, V. Karalekas, P. Harper, "High efficiency supercontinuum generation using ultra-long Raman fiber cavities," Opt. Express 17(20), pp. 17909-17915 (2009)

6. M. Alcón-Camas, A.E. El-Taher, H. Wang, P. Harper, V. Karalekas, J.A. Harrison, J.D. Ania-Castañón, “Longdistance soliton transmission through ultralong fiber lasers," Opt. Lett. 34(20), pp. 3104-3106 (2009)

7. J. Scheuer, A. Yariv, "Giant fiber lasers: a new paradigm for secure key distribution," Phys. Rev. Lett. 97(14), 140502 (2006)

8. B. Bristiel, S. Jiang, P. Gallion, E. Pincemin, "New model of noise Figure and RIN transfer in fiber Raman amplifiers," IEEE Photo. Tech. Lett. 18(8) , pp.980-982 (2006) 